\title{
In Vitro Susceptibility Testing of Silver and Zinc Nanoparticles on Different Fungal Species
}

\author{
MOSAAB SAAFAN, M.Sc.*; MOHAMMED FADL, M.D.*; MAHMOUD HASHESH, M.D.* and \\ MAHMOUD ALAASAR, Ph.D.** \\ The Department of Clinical Pathology, Faculty of Medicine* and The Regional Center for Mycology and Biotechnology, \\ Faculty of Science**, Al-Azhar University, Cairo, Egypt
}

\begin{abstract}
Background: Due to the dramatic increase of opportunistic fungal strains and infection, searching for new method to kill or even stop their growing becomes mandatory. Nanoparticles as clusters of atoms in the size of $1-100 \mathrm{~nm}$ is one of these method and it's what we use in this paper.
\end{abstract}

Aim of Study: From the current antifungal drugs, 3 of them were used in the present study. These were Itraconazole, Amphotercin B, and Terbinifine. Also, 2 metals in nanoparticles were used (silver and zinc oxide) to test the efficacy of these nanoparticles on different fungal strains. 16 fungal strains representing different fungal groups were tested.

Material and Methods: (1) Silver and zinc oxide nanoparticles. (2) Well identified 16 fungal strains. (3) Antifungal drugs (Itraconazole, Amphotercin B, and Terbinifine).

$\mathrm{Ag}$ and $\mathrm{Zn}$ oxide nanoparticles tested against 16 fungal strains to detect Minimal Inhibitory Concentration (MIC). Then, antifungal drugs (Itraconazole, Amphotercin B, and Terbinifine) tested against the same 16 fungal strains. Finally, combination between the nanoparticles and the antifungal drugs tested against the previous fungal strains.

Results: Comparison studies were performed on different fungal strains by using NPs, routine antifungal drugs, and combination between them. The most effective was the routine antifungal drugs then the combination and the weakest was the NPs.

Conclusions: Combination between NPs and antifungal drugs is promising and need more efforts to put it in practice.

Key Words: AgNPS - ZnO NPs - Antifungal drugs - Fungal infection.

\section{Introduction}

IN recent years, a rapid increase in microbes that are resistant to conventional antibiotics has been

Correspondence to: Dr. Mosaab Saafan, The Department of Clinical Pathology, Faculty of Medicine, Al-Azhar University, Cairo, Egypt observed [1]. Especially, the frequency of infections provoked by opportunistic fungal strains has increased dramatically. Even though the majority of invasive fungal infections are still due to the Aspergillus or Candida species, the spectrum of fungal pathogens has changed and diversified [2]. Azoles that inhibit sterol formation and polyenes that bind to mature membrane sterols have been the mainstays regarding antifungal therapy for several decades [3] . However, not only the emergence of fluconazole resistance among different pathogenic strains but also the high toxicity of amphotericin B [4] has prompted research on new antifungal agents [5]. We specifically regarded nanoparticles as clusters of atoms in the size of $1-100 \mathrm{~nm}$. 'Nano' is a Greek word synonymous to dwarf meaning extremely small. The use of nanoparticles is gaining impetus in the present century as they posses defined chemical, optical and mechanical properties. Among them, the metallic nanoparticles are most promising as they contain remarkable antimicrobial properties due to their large surface area to volume ratio, which is of interest to researchers due to the growing microbial resistance against metal ions, antibiotics, and the development of resistant strains $[6,7]$.

Different types of nanomaterials like copper, zinc, titanium [8], magnesium, gold [9], alginate [10] and silver have been developed but silver nanoparticles (Nano-Ag) have proved to be most effective as they exhibit potent antimicrobial efficacy against bacteria, viruses and eukaryotic microorganisms.

About Nano-Ag used as a disinfectant drug also has some risks as the exposure to silver can cause argyrosis or argyria; it can be toxic to mammalian cells [7]. 
The current investigation supports the theory that the use of silver ions or metallic silver as well as Nano-Ag can be exploited in medicine for burn treatment, dental materials, coating stainless steel materials, textile fabrics, water treatment, sunscreen lotions, etc. and posses low toxicity to human cells, high thermal stability and low volatility [11]. Many studies have shown the biological effects of Nano$\mathrm{Ag}$, however, its effects against fungal pathogens have not yet been fully studied.

Recent studies have demonstrated antimicrobial activities of various NP materials, including silver [12], copper [13], titanium dioxide [14], and zinc oxide [15]. Highly ionic nanoparticulate metal oxides such as zinc oxide nanoparticles (ZnO NPs) are unique in that they can be produced with high surface areas and with unusual crystal structures [16] compared to organic materials, inorganic materials such as $\mathrm{ZnO}$ possess superior durability, greater selectivity, and heat resistance. Moreover, zinc is a mineral element essential to human health and $\mathrm{ZnO}$ is a form in the daily supplement for zinc. $\mathrm{ZnO}$ NPs also have good biocompatibility to human cells. The antibacterial and antifungal activity of bulk $\mathrm{ZnO}$ powders has been demonstrated already.

\section{Material and Methods}

The practical part of this paper was done at the Regional Center for Mycology and Biotechnology, Faculty of Science, Al-Azhar University in February and March 2016.

\section{Material:}

1- Silver and zinc oxide nanoparticles.

2- Well identified 16 fungal strains.

3- Antifungal drugs (Itraconazole, Amphotercin $\mathrm{B}$, and Terbinifine).

\section{Methods:}

$\mathrm{Ag}$ and $\mathrm{Zn}$ oxide nanoparticles tested against 16 fungal strains to detect Minimal Inhibitory Concentration (MIC). Then, antifungal drugs (Itraconazole, Amphotercin B, and Terbinifine) tested against the same 16 fungal strains. Finally, combination between the nanoparticles and the antifungal drugs tested against the previous fungal strains.

\section{Results}

Table (1): Antifungal Activity as MICS ( $\mu \mathrm{g} / \mathrm{ml}$ ) of NPs and Itraconazole against tested microorganisms.

\begin{tabular}{|c|c|c|c|c|c|}
\hline \multirow[b]{2}{*}{ Tested microorganisms } & Ag Nps & Zn Nps & Itraconazole & $\begin{array}{l}\text { Itraconazole } \\
+\mathrm{Ag} \text { Nps }\end{array}$ & $\begin{array}{c}\text { Itraconazole } \\
+ \text { Zn Nps }\end{array}$ \\
\hline & \multicolumn{5}{|c|}{ Minimum inhibitory concentration ( $\mathrm{g} / \mathbf{m}$ ) } \\
\hline \multicolumn{6}{|l|}{ FUNGI: } \\
\hline Aspergillus fumigatus (RCMB 02568) & 32 & 16 & 8 & 8 & 2 \\
\hline Aspergillus niger (RCMB 02724) & 64 & 16 & 8 & 8 & 1 \\
\hline Aspergillus terreus (RCMB 02574) & 16 & 8 & 16 & 16 & 4 \\
\hline Aspergillus flavus (RCMB 02782) & 32 & 8 & 8 & 8 & 4 \\
\hline Aspergillus clavatus (RCMB 02791) & 16 & 16 & 8 & 8 & 2 \\
\hline Candida albicans (RCMB 05036) & 64 & 16 & 16 & 4 & 16 \\
\hline Candida tropicalis (RCMBA 05239 ) & 32 & 8 & 4 & 1 & 4 \\
\hline Candida parapsilosis (RCMBA 05237 ) & 32 & 16 & 2 & 1 & 2 \\
\hline Cryptococcus neoformans (RCMB 05642) & 128 & 256 & 32 & 16 & 32 \\
\hline Geotricum candidum (RCMB 05097) & 8 & 4 & 2 & 1 & 2 \\
\hline Trichosporon beigelii (RCMB 07624) & 64 & 128 & 64 & 64 & 64 \\
\hline Penicillium expansum (RCMB 01924) & 32 & 64 & 16 & 16 & 16 \\
\hline Syncephalastrum racemosum (RCMB 05922) & 16 & 8 & 4 & 4 & 4 \\
\hline Trichophyton rubrum (RCMB 0938) & 256 & 128 & 64 & 64 & 64 \\
\hline Microsporum canis (RCMB 0834) & 64 & 32 & 32 & 32 & 32 \\
\hline Trichophyton mentagrophytes (RCMB 0925) & 128 & 128 & 64 & 64 & 64 \\
\hline
\end{tabular}


Table (2): Antifungal activity as MICS ( $\mathrm{g} / \mathrm{l}$ ) of NPs and Amphotercin B against tested microorganisms.

\begin{tabular}{|c|c|c|c|c|c|}
\hline \multirow{2}{*}{ Tested microorganisms } & $\mathrm{Ag} \mathrm{Np}$ & $\mathrm{Zn} \mathrm{Nps}$ & $\begin{array}{c}\text { Amphotericin } \\
\text { B }\end{array}$ & $\begin{array}{l}\text { Amphotericin } \\
\text { B + Ag Nps }\end{array}$ & $\begin{array}{c}\text { Amphotericin } \\
\text { B + Zn Nps }\end{array}$ \\
\hline & \multicolumn{5}{|c|}{ Minimum inhibitory concentration ( $\mathrm{g} / \mathrm{m}$ ) } \\
\hline \multicolumn{6}{|l|}{ FUNGI: } \\
\hline Aspergillus fumigatus (RCMB 02568) & 32 & 16 & 16 & 8 & 16 \\
\hline Aspergillus niger (RCMB 02724) & 64 & 16 & 4 & 0.5 & 4 \\
\hline Aspergillus terreus (RCMB 02574) & 16 & 8 & 4 & 1 & 4 \\
\hline Aspergillus flavus (RCMB 02782) & 32 & 8 & 32 & 4 & 16 \\
\hline Aspergillus clavatus (RCMB 02791) & 16 & 16 & 16 & 2 & 16 \\
\hline Candida albicans (RCMB 05036) & 64 & 16 & 4 & 4 & 4 \\
\hline Candida tropicalis (RCMBA 05239 ) & 32 & 8 & 8 & 8 & 8 \\
\hline Candida parapsilosis (RCMBA 05237 ) & 32 & 16 & 8 & 8 & 8 \\
\hline Cryptococcus neoformans (RCMB 05642) & 128 & 256 & 16 & 16 & 16 \\
\hline Geotricum candidum (RCMB 05097) & 8 & 4 & 4 & 4 & 1 \\
\hline Trichosporon beigelii (RCMB 07624) & 64 & 128 & 128 & 32 & 128 \\
\hline Penicillium expansum (RCMB 01924) & 32 & 64 & 8 & 8 & 8 \\
\hline Syncephalastrum racemosum (RCMB 05922) & 16 & 8 & 2 & 2 & 1 \\
\hline Trichophyton rubrum (RCMB 0938) & 256 & 128 & 16 & 16 & 16 \\
\hline Microsporum canis (RCMB 0834) & 64 & 32 & 8 & 8 & 8 \\
\hline Trichophyton mentagrophytes (RCMB 0925) & 128 & 128 & 32 & 32 & 32 \\
\hline
\end{tabular}

Table (3): Antifungal activity as MICS ( $\mathrm{g} / \mathrm{l}$ ) of NPs and terbinafine against tested microorganism.

\begin{tabular}{|c|c|c|c|c|c|}
\hline & Ag Nps & Zn Nps & Terbinafine & $\begin{array}{l}\text { Terbinafine } \\
+ \text { Ag Nps }\end{array}$ & $\begin{array}{c}\text { Terbinafine } \\
+ \text { Zn Nps }\end{array}$ \\
\hline & \multicolumn{5}{|c|}{ Minimum inhibitory concentration ( $\mathrm{g} / \mathrm{ml}$ ) } \\
\hline \multicolumn{6}{|l|}{ FUNGI: } \\
\hline Aspergillus fumigatus (RCMB 02568) & 32 & 16 & 4 & 2 & 4 \\
\hline Aspergillus niger (RCMB 02724) & 64 & 16 & 2 & 1 & 2 \\
\hline Aspergillus terreus (RCMB 02574) & 16 & 8 & 2 & 1 & 2 \\
\hline Aspergillus flavus (RCMB 02782) & 32 & 8 & 2 & 0.5 & 2 \\
\hline Aspergillus clavatus (RCMB 02791) & 16 & 16 & 4 & 1 & 4 \\
\hline Candida albicans (RCMB 05036) & 64 & 16 & 64 & 64 & 64 \\
\hline Candida tropicalis (RCMBA 05239 ) & 32 & 8 & 128 & 128 & 128 \\
\hline Candida parapsilosis (RCMBA 05237 ) & 32 & 16 & 128 & 128 & 128 \\
\hline Cryptococcus neoformans (RCMB 05642) & 128 & 256 & 256 & 256 & 256 \\
\hline Geotricum candidum (RCMB 05097) & 8 & 4 & 32 & 32 & 32 \\
\hline Trichosporon beigelii (RCMB 07624) & 64 & 128 & 64 & 64 & 64 \\
\hline Penicillium expansum (RCMB 01924) & 32 & 64 & 4 & 1 & 4 \\
\hline Syncephalastrum racemosum (RCMB 05922) & 16 & 8 & 2 & 2 & 2 \\
\hline Trichophyton rubrum (RCMB 0938) & 256 & 128 & 8 & 8 & 8 \\
\hline Microsporum canis (RCMB 0834) & 64 & 32 & 4 & 4 & 4 \\
\hline Trichophyton mentagrophytes (RCMB 0925) & 128 & 128 & 8 & 8 & 8 \\
\hline
\end{tabular}

\section{Discussion}

Our study is compatible with:

- Bonilla, [17] demonstrated that A. Flavus isolates were inhibited to various extents by different concentrations of silver nanoparticles and showed significant inhibition effectiveness reached to 94\% with $\mathrm{ZnO}-\mathrm{NPs}$.
- Chan, [18] showed that using nanosilver suspension at the concentration of 50ppm inhibits the growth of Aspergillus niger by $70 \%$.

- Kim et al., [19] reported significant antifungal activity of silver nanoparticles against various strains of Candida albicans, C. tropicalis, C. glabrata, C. parapsilosis, C. krusei and Trichophyton menta-grophytes. 
Our study is not compatible with:

- Selvaraj et al., [20] showed that the AgNPs suspension was an effective growth inhibition factor against Aspergillus niger.

- Sardi et al., [21] revealed that AgNPs exhibited potent activity against clinical isolates Trichophyton mentagrophytes and Candida species.

\section{Conclusions:}

- Regarding Filamentous fungi, Terbinafine combined with AgNPs is the best choice.

- Regarding yeast and yeast like fungi, itraconazole combined with Ag Nps is the best choice.

- Regarding dermatophytes, Terbinafine, Terbinafine combined with AgNps OR Terbinafine combined with $\mathrm{ZnO}$ NPs are the best choice.

\section{Recommendations:}

- Continuous support and frequent observation is required for such a program to be successful.

- Concern has been raised on the toxicity of chemical agents used in AgNPs synthesis. Thus, it is essential to develop a green approach for AgNPs production without using hazardous substances to the human health and environment.

- Compared with the traditional synthetic methods, biological systems provide a novel idea for the production of nano-materials.

\section{References}

1- GOFFEAU A.: Drug resistance: The fight against fungi. Nature, 452 (7187): 541-2, 2008.

2- DENNING D.: Epidemiology and pathogenesis of systemic fungal infections in the immunocompromised host. J. Antimicrob. Chemother., 28 (B): 1-16, 1991.

3- KULLBERG B. and De PAUW B.: Therapy of invasive fungal infections. Neth. J. Med., 55 (3): 118-27, 1999.

4- ALEXANDER B. and PERFECT J.: Antifungal resistance trends towards the year 2000. Implications for therapy and new approaches. Drugs, 54 (5): 657-78, 1997.

5- KONTOYIANNIS D., MANTADAKIS E. and SAMONIS G.: Systemic mycoses in the immunocompromised host: An update in antifungal therapy. J. Hosp. Infect., 53 (4): 243-58, 2003

6- RAI M., YADAV A. and GADE A.: Silver nanoparticles as a new generation of antimicrobials. Biotechnol. Adv., 27 (1): 76-83, 2009.

7- GONG P., LI H., HE X., WANG K., HU J., ZHANG S. and YANG X.: Preparation and antibacterial activity of $\mathrm{Fe} 3 \mathrm{O} 4$ at $\mathrm{Ag}$ nanoparticles. Nanotechnology, 18 (28): 604-11, 2007.

8- SCHABES-RETCHKIMAN P., CANIZAL G., HERRERA-BECERRA R., ZORRILLA C., LIU H. and ASCEN-
CIO J.: Biosynthesis and characterization of Ti/Ni bimetallic nanoparticles. Opt. Mater., 29 (1): 95-9, 2006.

9- GU H., HO P., TONG E., WANG L. and XU B.: Presenting vancomycin on nanoparticles to enhance antimicrobial activities. Nano. letters., 3 (9): 1261-3, 2003.

10- AHMAD Z., PANDEY R., SHARMA S. and KHULLER G.: Alginate nanoparticles as antituberculosis drug carriers: Formulation development; pharmacokinetics and therapeutic potential. Indian J. Chest Dis. Allied. Sci., 48 (3): 171-6, 2006.

11- DURÁN N., MARCARTO P., De SOUZA G., ALVES O. and ESPOSITO E.: Antibacterial effect of silver nanoparticles produced by fungal process on textile fabrics and their effluent treatment. J. Biomed. Nanotechnol., 3 (2): 203-8, 2007.

12- KIM K., SUNG W., MOON S., CHOI J., KIM J. and LEE D.: Antifungal effect of silver nanoparticles on dermatophytes. J. Microbiol. Biotechnol., 18 (8): 1482-4, 2008.

13- CIOFFI N., TORSI L., DITARANTO N., TANTILLO G., GHIBELLI L. and SABBATINI L.: Copper nanoparticle/polymer composites with antifungal and bacteriostatic properties. Chem. Mater., 17 (21): 5255-62, 2005.

14- KWAK S., KIM S. and KIM S.: Hybrid organic/inorganic reverse osmosis (RO) membrane for bactericidal antifouling Preparation and characterization of $\mathrm{TiO}_{2}$ nanoparticle self-assembled aromatic polyamide thinfilm-composite (TFC) membrane. Environ. Sci. Technol., 35 (11): 238894, 2001.

15- LIU Y., HE L., MUSTAPHA A., LI H. and LIN M.: Antibacterial activities of zinc oxide nanoparticles against Escherichia coli O157: H7. J. Appl. Microbiol., 107 (4): 1193-201, 2009.

16- KLABUNDE K., STARK J., KOPER O., MOHS C., PARK D. and DECKER S.: Nanocrystals as stoichiometric reagents with unique surface chemistry. J. Phys. Chem., 100: 12142-53, 1996.

17- BONILLA J., GUERRERO D., SUAREZ C., LÓPEZ C. and SAEZ R.: In vitro antifungal activity of silver nanoparticles against fluconazole-resistant Candida species. World J. Microbiol. Biotechnol., 31 (11): 1801-9, 2014.

18- CHAN Y. and DON M.: Characterization of Ag Nanoparticles Produced by White-Rot Fungi and Its in vitro Antimicrobial Activities. International Arabic Journal of Antimicrobial Agents., 2 (3): 8 pages, 2012.

19- KIM K., SUNG W., SUH B., MOON S., CHOI J. and KIM J.: Antifungal activity and mode of action of silver nano-particles on Candida albicans. Biometals, 22 (2): 235-24, 2008

20- SELVARAJ M., PANDURANGAN P., RAMASAMI N., RAJENDRAN S., SANGILIMUTHU S. and PERUMAL P.: Highly Potential Antifungal Activity of QuantumSized Silver Nanoparticles Against Candida albicans. Appl. Biochem. Biotechnol., 173 (1): 55-66, 2014.

21- SARDI J., SCORZONI L., BERNARDI T., FUSCOALMEIDA A. and GIANNINI M.: Candida species: Current epidemiology pathogenicity, biofilm formation, natural antifungal products and new therapeutic options. J. Med. Microbiol., 62: 10-24, 2013. 


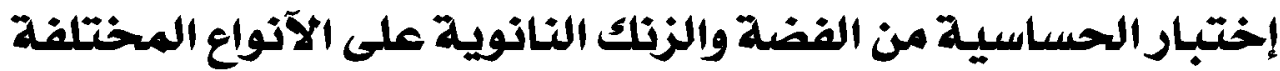 من الفطريات فى المختبر الفرئر}

يعد النانومتر آحد وحدات قياس الآطوال ويساوى جزء من المليار من المتر وهو مشتق من الكلمة اليونانية التى تعنى المثناهى الصغر.

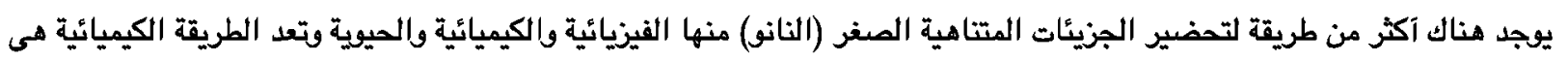

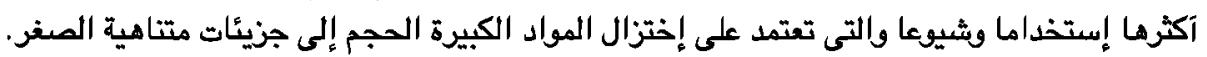

تتميز جزئات الفضة المتناهية الصغر والتى يتراوح طولها ما بين ا- - .1 نانو متر بالقدرة على مكافحة البكتريا بنوعيها الموجبة والسالبة لصبغة الجرام وكذلك الفطريات المختفة. الهدف من البحث: دراسة مقارتة بين جزيئات النانو للفضدة وآكسيد الزنك ومضادات الفطريات الثائعة الإستخدام. الآدوات وطرق البحث: الآدوات:

$$
\begin{aligned}
& \text { 1- جزيئات النانو للفضدة وآكسيد الزنك. } \\
& \text { r- عينات معرفة سابقا للفطريات. } \\
& \text { ب - مضادات فطريات ثـائعة الإستخدام. }
\end{aligned}
$$

طرق البحث: عن طريق عينات معرفة سـابقا لفطريات ومقارنة مضادات شـائعة الإستضدام مع جزئًات النانو للفضة وآكسيد الزنك كمضادات لهذه الفطريات.

ملخص نتائج الدراسة: • أوضحت هذه الدراسة كفاءة عالية من جزيئات الفضة وآكسيد الزتك متناهيا الصغر على الآنقاع المختفة من الفطريات.

• وآوضحت آيضا آن جزيئات الفضة وآكسيد الزنك منتاهيا الصغر آعطت آفضل النتائج بعد خلطها مع مضادات الفطريات الروتينية. وقد آوصى البحث بدراسة جزيئات الفضدة وآكسيد الزنك متناهيا الصغر عند خلطها مع بعضها البعض بالإضافة إلى مضادات الفطريات 